\title{
A Walk in the Park: Perceptions of Place through Mapping
}

A Walk in the Park is the initial stage of an exploratory mapping project that investigates the aesthetics of cartography and its capacity to tell stories, communicate ideas, and evoke sensory impression and experience. This yearlong exercise explores the Edinburgh Gardens, a 60acre parkland situated in the heart of North Fitzroy, an inner suburb just north of the city of Melbourne, Australia. The focus of this project is the perception of place, the transitory nature of that perception, and the role maps might play in enhancing that perception. I wanted to draw upon my diverse experiences of the park to visually explore cartography's potential to arouse the senses, evoke emotion, and stimulate the imagination.

The role of time emerged with each park visit and I drew upon Torsten Hägerstrand's concept of a space-time cube as a method for investigation. A space-time cube is a graphic visualization combining time and space within a contained three-dimensional space, a "cube." During each walk, I collected data that informed

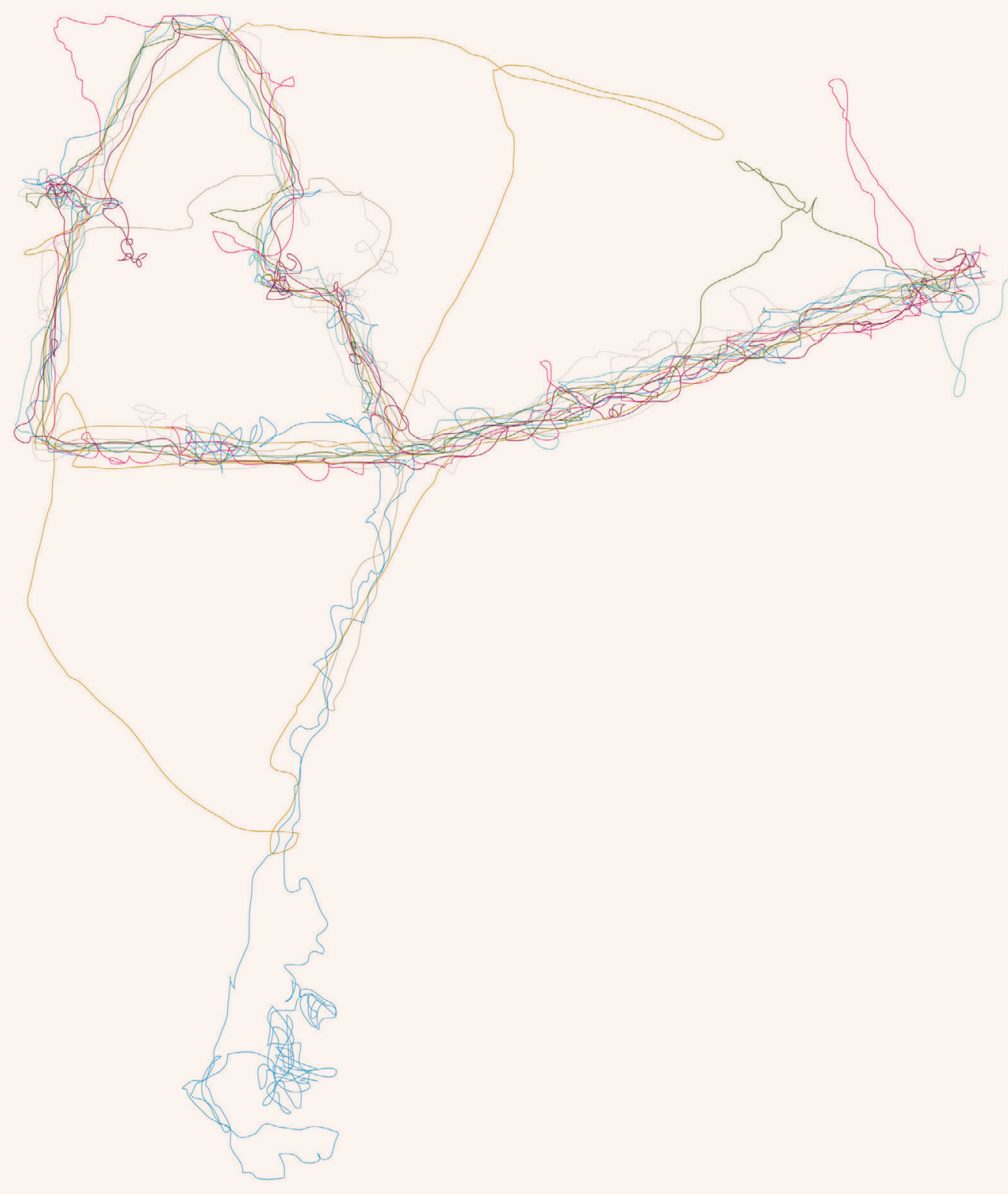

Figure 1. MAPPED ROUTES, 2017. Paths undertaken during walks.

my own interpretation of space-time cubes, each one representing a different walk (Figure 1). I compared the route and duration of the walks with the weather and with 

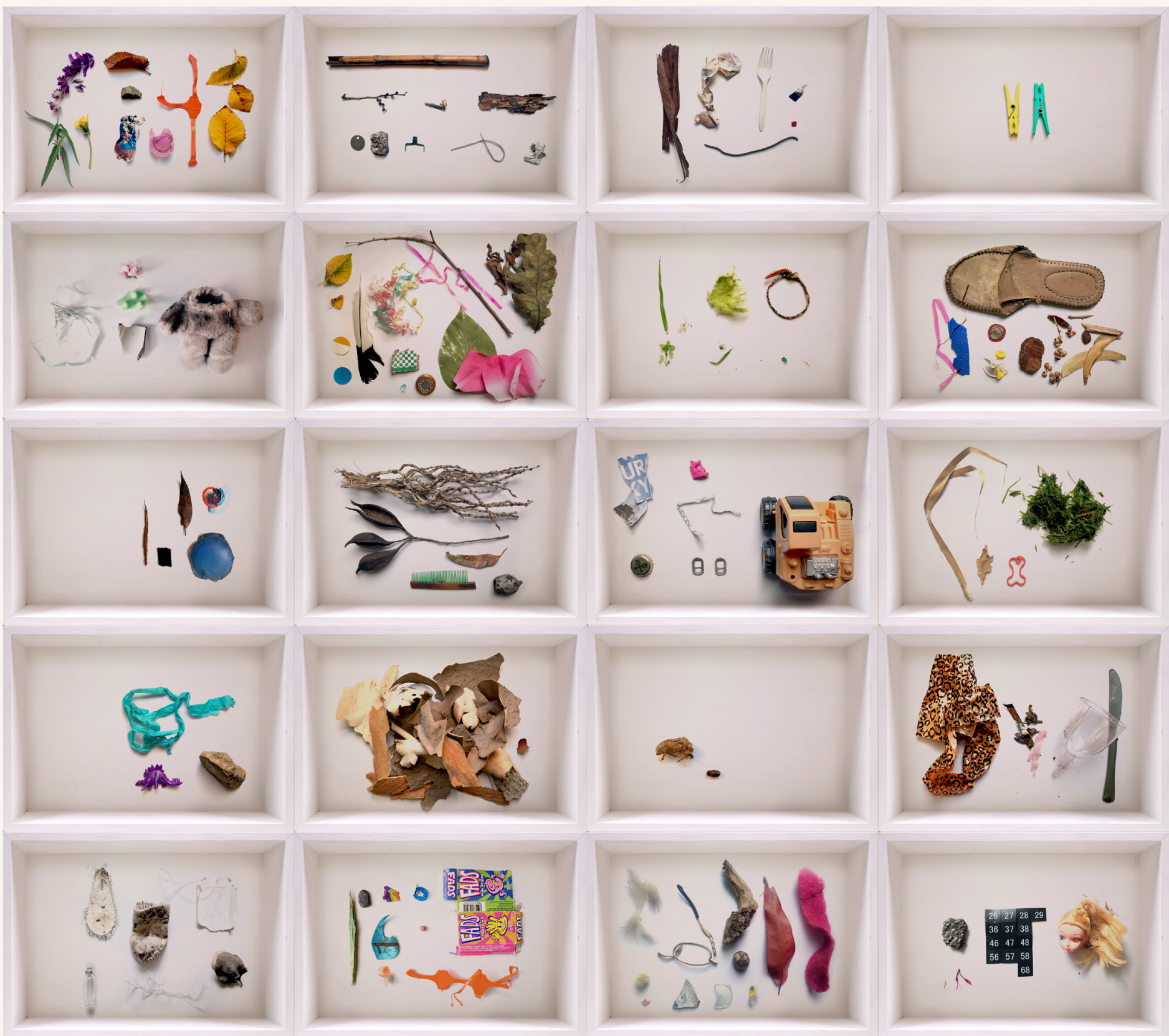

Figure 2. CABINET OF CURIOSITIES, 2017. Selections of found objects from each walk.

photographs I had taken. From this information, I generated colour palettes through sampling dominant colours of the photographs and then began a "cabinet of curiosities" showcasing a collection of objects I found during each visit (Figures 2-5). To fully capture the experience and my perceptions of the walks, I wanted to use a combination of the collected data. I selectively filtered the information, choosing aspects that best embodied the experience of each walk. As I investigated how to put these disparate elements back together, I reassembled and reinterpreted fragments pulled from the already filtered information, which then collectively formed the basis of my individual space-time cubes (Figure 6).

A sculptural project appeared to be a suitable method for exploring the potential of the three-dimensional spacetime cube. Ice became my medium of choice, as I wanted one that allowed for the suspension of the found artifacts 

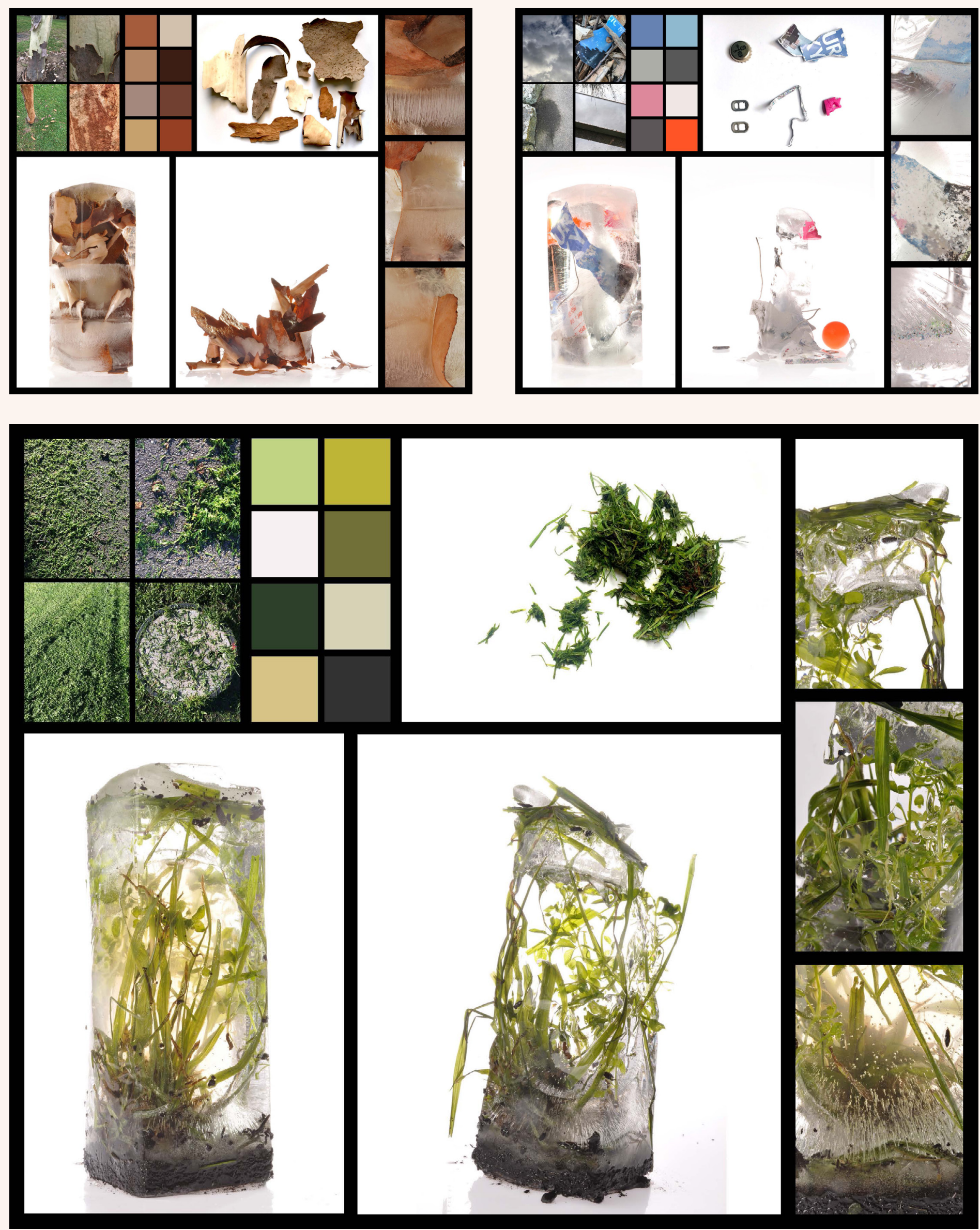

Figures 3-5. December (top left), November (top right), and August (bottom) collections and colour palettes. 
from my walks. Ice also proved to be an excellent medium for exploring weight and light, by manipulating its constitution with texture and adding colour with pigments. Ice allowed me to work in layers and intervene in its freezing at different points to build my space-time cubes. The transitory nature of ice as a medium was also important in capturing the transitory nature of time. My walks, and the perception and experience of the walks, were temporary,
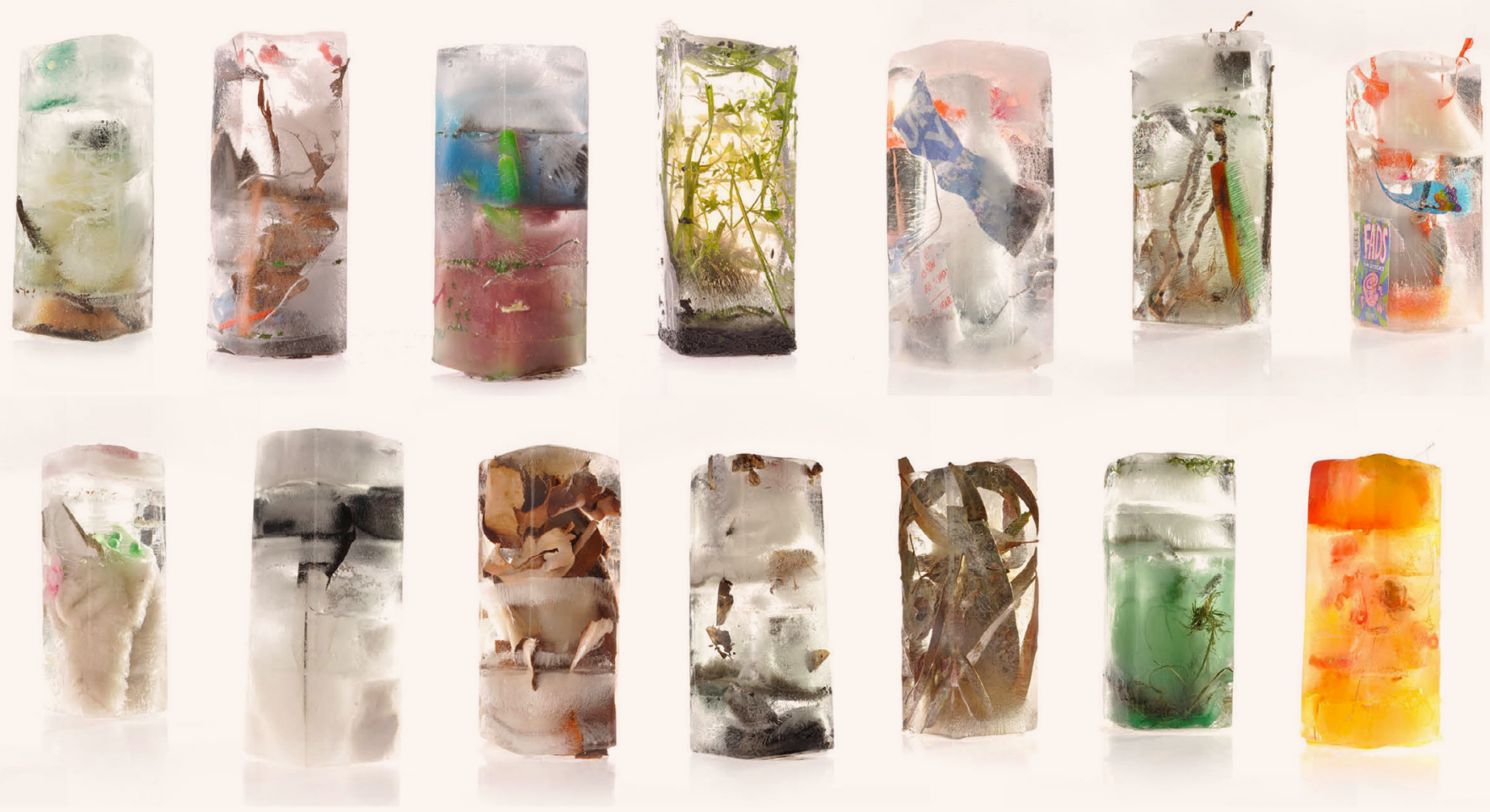

Figure 6. Space-time cubes.
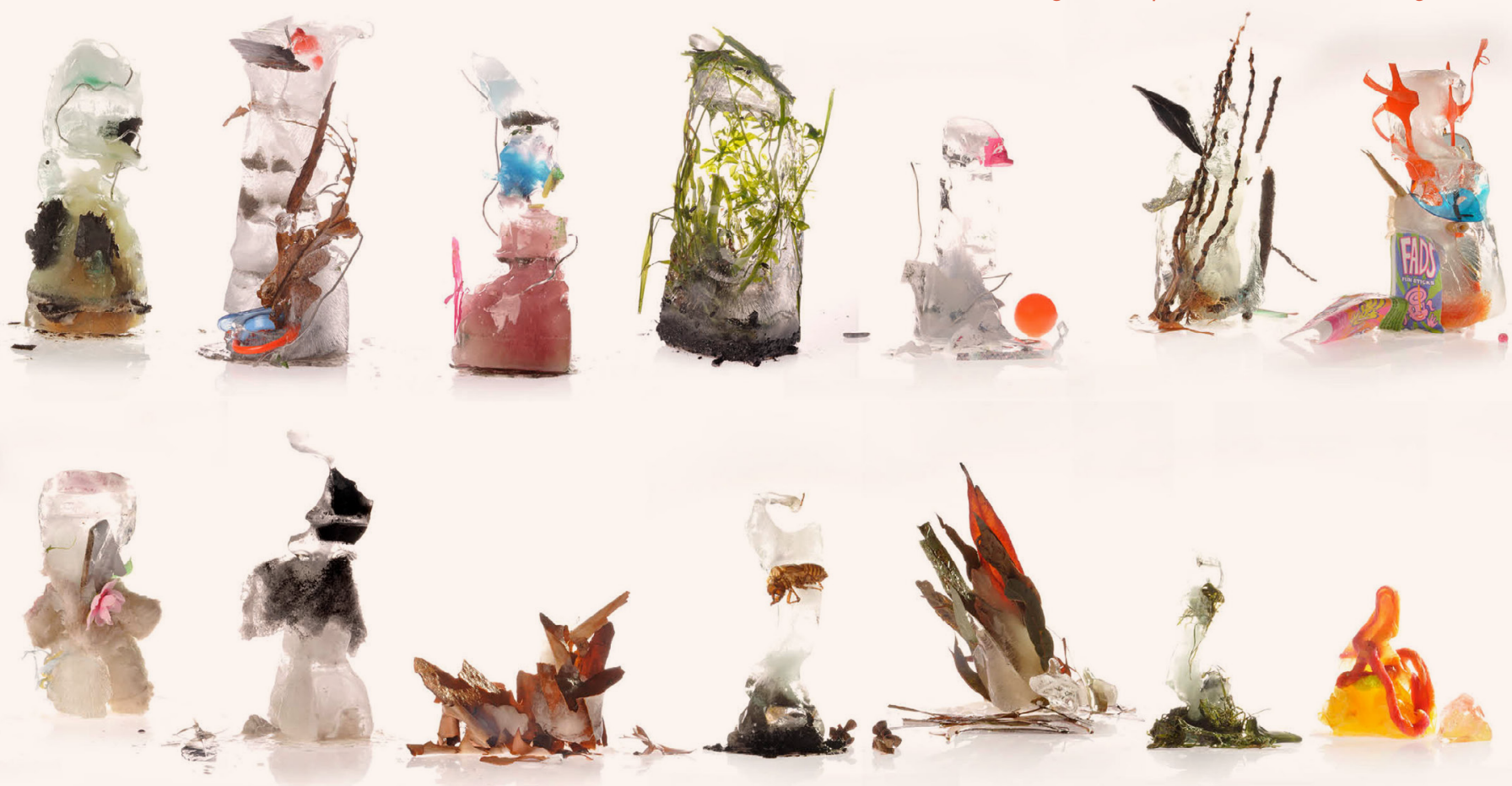
thus freezing a moment of time seemed fitting. So, it is through ice that I explored the concept of Hägerstrand's space-time cubes for each walk in the park.

The melting of the space-time cubes represents time passing, embodying the temporal aspect of each walk (Figure 7). As the ice melts, it reveals layers of the walk, exposing materials and found artifacts. This reveal is reflective of the walk itself that bares its milieu to you as you walk the path. With melting, the colour palettes and light continually shift, similar to clouds passing overhead on a sunny day, or an unexpected encounter, or sudden change in mood. The melting illuminates those personal moments, emotions, or secrets embedded within each walk (see video).

When one visits the park, one just passes through. One's time in the park is transient, and the only clue that one was ever there are the traces left behind. When the space-time cube has melted, the walk is complete and only the found artifacts remain (Figure 8).

Visual Fields focuses on the appreciation of cartographic aesthetics and design, featuring examples of inspirational, beautiful, and intriguing work. Suggestions of works that will help enhance the appreciation and understanding of the cartographic arts are welcomed, and should be directed to Section Editor Matt Dooley: mapdooley@gmail.com

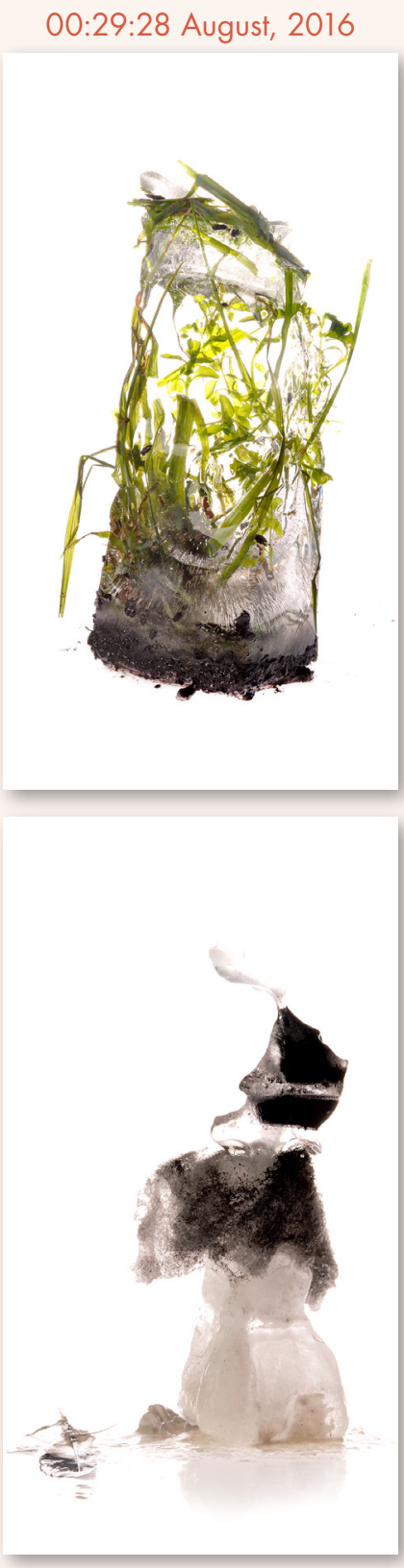

00:34:51 May, 2017
01:21:05 July, 2016
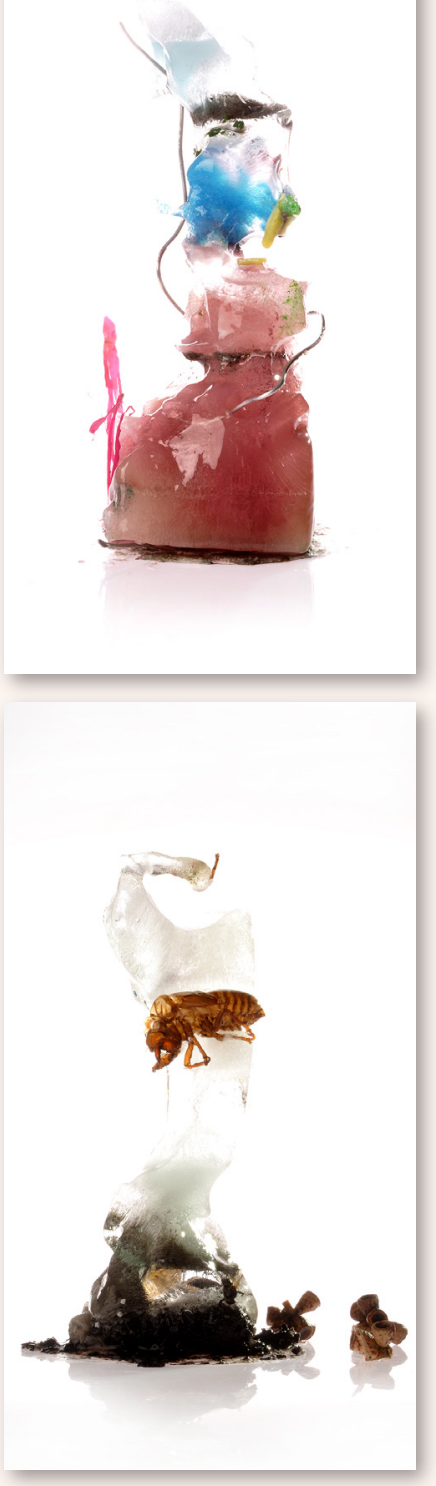

00:20:38 February, 2017
01:01:00 January, 2017
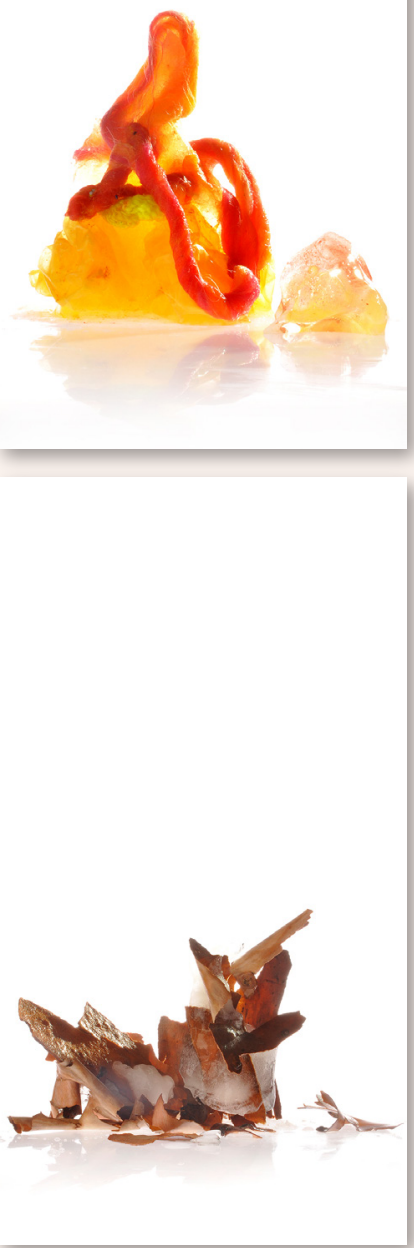

00:23:57 December, 2016
Figure 8. TRANSITORY NATURE OF TIME. Large scale digital prints on paper. 